\title{
On invertible generating pairs of fundamental groups of graph manifolds
}

\author{
MiCHEL BOILEAU \\ RICHARD WEIDMANN
}

\begin{abstract}
We study invertible generating pairs of fundamental groups of graph manifolds, that is, pairs of elements $(g, h)$ for which the map $g \mapsto g^{-1}, h \mapsto h^{-1}$ extends to an automorphism. We show in particular that a graph manifold is of Heegaard genus 2 if and only if its fundamental group has an invertible generating pair.
\end{abstract}

20F34; 57M27

Dedicated to Heiner Zieschang, our teacher, mentor and friend

\section{Introduction}

Let $M$ be a closed orientable irreducible 3-manifold. We say that $M$ is a 2-generated manifold if $G=\pi_{1}(M)$ can be generated by two elements. Thurston asked the following question (see also Kirby [14, Problem 3.15A]):

Question 1 Given a generating pair $(g, h)$ for $G=\pi_{1}(M)$, is there an automorphism $\alpha: G \rightarrow G$ such that $\alpha(g)=g^{-1}$ and $\alpha(h)=h^{-1}$ ?

We call such an automorphism $\alpha$ an inversion of $\pi_{1}(M)$, and if it exists we say that the generating pair $(g, h)$ is invertible. Note that 3-manifolds of Heegaard genus 2 always have inversions. We say that $\alpha$ is a hyperelliptic inversion if $\alpha$ is induced by the hyperelliptic involution of a genus 2 Heegaard splitting.

Question 2 Is any inversion of $\pi_{1}(M)$ hyperelliptic?

For 2-generated hyperbolic manifolds Question 1 has a positive answer by Jorgensen's construction (see Adams [1] and Thurston [20, Chapter 5]) while Question 2 is still open. In this article we consider the case of graph manifolds. Our main result is the following: 
Theorem 3 A closed orientable graph manifold $M$ has Heegaard genus two if and only if $\pi_{1}(M)$ admits an inversion.

As there are graph manifolds and even Seifert manifolds with 2-generated fundamental group that do not admit a genus 2 Heegaard splitting (see Boileau and Zieschang [6] and Weidmann [21]) we get a negative answer to Question 1:

Corollary 4 There exist graph manifolds with 2-generated fundamental group whose fundamental groups have no invertible generating pair.

In the case of Seifert manifolds we have a stronger result, namely:

Theorem 5 Let $M$ be a closed orientable Seifert 3-manifold. Then any inversion of $\pi_{1}(M)$ is hyperelliptic.

For Seifert 3-manifolds this gives a positive answer to Question 2. We believe that, using the same methods, this result can be extended more generally to graph manifolds.

Even in the case of Seifert manifolds, it turns out that non-invertible generating pairs are rather plentiful and also many 3-manifolds of genus 2 have such generating pairs. For example Heegaard genus 2 Seifert manifolds with base $S^{2}$ and three singular fibers of order $\{2,3, r\}$ such that $\operatorname{gcd}(6, r)=1$ and $r \geq 7$ have infinitely many generating pairs which are not invertible. The fundamental group of some of these examples are 2-generated subgroups of $\widetilde{P S L}(2, \mathbb{R})$.

It should be remarked also that Theorem 5 does not imply that any invertible generating pair of the fundamental group of a Seifert manifold is geometric, where we say that a generating pair is geometric if it corresponds to the spine of a handlebody of a genus 2 splitting of the manifold. In fact there are examples of invertible generating pairs that are seemingly unrelated to the geometric generating pair that induces the same inversion. The situation seems most mysterious for the Brieskorn homology sphere wich is a Seifert manifold with base space $S^{2}(2,3,7)$. It turns out that its fundamental group admits an invertible generating pair which is not geometric after Nielsen equivalence and passing to roots.

\section{Preliminaries}

Let $G$ be a group and $(g, h)$ be a generating pair of $G$. If the map $\bar{\alpha}(g)=g^{-1}$ and $\bar{\alpha}(h)=h^{-1}$ extends to an automorphism $\alpha$ of the group $G$ then we say that the 
generating pair $(g, h)$ induces an inversion $\alpha$ of $G$, sometimes we call $\alpha$ the inversion relative $(g, h)$. We then further say that the generating pair $(g, h)$ is invertible.

It is clear that for a given group some generating pair might induce an inversion while another does not. In the following however we will see that this property is an invariant of the Nielsen equivalence class.

Recall that two tuples $T=\left(g_{1}, \ldots, g_{n}\right)$ and $T^{\prime}=\left(h_{1}, \ldots, h_{n}\right)$ are elementary equivalent if one of the following holds:

(1) $h_{i}=g_{\sigma(i)}$ for $1 \leq i \leq n$ and some $\sigma \in S_{n}$.

(2) $h_{1}=g_{1}^{-1}$ and $h_{i}=g_{i}$ for $i \geq 2$.

(3) $h_{1}=g_{1} g_{2}$ and $h_{i}=g_{i}$ for $i \geq 2$.

We further say that two tuples $T$ and $T^{\prime}$ are Nielsen equivalent if there is a sequence of tuples $T=T_{0}, T_{1}, \ldots, T_{k-1}, T_{k}=T^{\prime}$ of tuples such that $T_{i-1}$ and $T_{i}$ are elementary equivalent for $1 \leq i \leq k$.

Lemma 6 Let $G$ be a group and $(g, h)$ and $\left(g^{\prime}, h^{\prime}\right)$ be Nielsen-equivalent generating pairs. Then $(g, h)$ is invertible if and only if $\left(g^{\prime}, h^{\prime}\right)$ is invertible.

Proof It clearly suffices to verify the assertion for pairs $(g, h)$ and $\left(g^{\prime}, h^{\prime}\right)$ that are elementary equivalent. If they are related as in (1) or (2) above then the assertion is obvious as the maps $\bar{\alpha}$ are defined the same way.

Thus we can assume that $g^{\prime}=g h$ and $h^{\prime}=h$. Suppose that $\alpha_{1}: G \rightarrow G$ is an inversion that extends the map $\bar{\alpha}_{1}:\{g, h\} \rightarrow G$ given by $\bar{\alpha}_{1}(g)=g^{-1}$ and $\bar{\alpha}_{1}(h)=h^{-1}$. We have to show that the map $\bar{\alpha}_{2}:\{g h, h\} \rightarrow G$ given by $\bar{\alpha}_{2}(g h)=h^{-1} g^{-1}$ and $\bar{\alpha}_{2}(h)=h^{-1}$ extends to an automorphism $\alpha_{2}: G \rightarrow G$, it is easily verified that this map $\alpha_{2}$ can be defined as $\alpha_{2}(x)=h^{-1} \alpha_{1}(x) h$ for all $x \in G$. This map is clearly an automorphism of $G$ and its restriction to $\{g h, h\}$ is $\bar{\alpha}_{2}$.

Occasionally we will change a generating pair in such a way that we replace one generator with a root. This operation also turns out to be well behaved:

Lemma 7 Let $G$ be a group, $g, h \in G$ and $n \geq 2$ such that $(g, h)$ and $\left(g^{n}, h\right)$ are generating pairs. Suppose that $(g, h)$ induces an inversion. Then $\left(g^{n}, h\right)$ induces the same inversion.

Proof This is immediate as $\left(g^{n}, h\right)$ is a generating pair and the induced inversion $\alpha$ must map $g^{n}$ to $\alpha\left(g^{n}\right)=\alpha(g)^{n}=\left(g^{-1}\right)^{n}=g^{-n}$. 
The following corollary is straightforward consequence of the proofs of Lemma 6 and Lemma 7.

Corollary 8 Let $\left(g^{\prime}, h^{\prime}\right)$ be an invertible generating pair of a group $G$ obtained from a generating pair $(g, h)$ by Nielsen equivalence and replacing $g$ and/or $h$ with roots. Then $(g, h)$ is also invertible and the inversions $\alpha$ induced by $(g, h)$ and $\alpha^{\prime}$ induced by $\left(g^{\prime}, h^{\prime}\right)$ give the same element in $\operatorname{Out}(G)$ (the outer automorphism group of $G$ ).

If $G$ is the fundamental group of a 3-manifold $M$ then there are situations when we can guarantee that a generating pair induces an inversion. If $M$ is a hyperbolic 3-manifold then the involution exists for any generating pair, this result is due to Jorgensen, see Adams [1] or Thurston [20, Chapter 5].

Another situation where we can guarantee the existence of the inversion is the case of geometric generating pairs: Suppose that the manifold $M$ is of Heegaard genus 2, that is, that $M=V_{1} \cup_{S} V_{2}$ where $V_{1}$ and $V_{2}$ are handlebodies of genus 2 glued together along their boundaries. Now $\pi_{1}\left(V_{i}\right)$ is free of rank 2 and the homomorphism $i_{*}: \pi_{1}\left(V_{i}\right) \rightarrow \pi_{1}(M)$ induced by the inclusion map is surjective. This implies that any generating pair $(g, h)$ of $\pi_{1}\left(V_{i}\right)$ gets mapped onto a generating pair $\left(i_{*}(g), i_{*}(h)\right)$ of $\pi_{1}(M)$. We call generating pairs that occur this way geometric generating pairs.

It has been shown by Birman and Hilden [3] that any geometric generating set induces an inversion. To see this it suffices to show that the hyperelliptic involution of the handlebody of genus 2 induces an automorphism of the fundamental group that maps the generators to their inverses and that this involution can be extended to the complementary handlebody. We call an inversion of $\pi_{1}(M)$ that is obtained as above a hyperelliptic inversion of $\pi_{1}(M)$. As any generating pair of $\pi_{1}(M)$ that is Nielsen equivalent to a generating pair that corresponds to the spine of handlebody in $M$ also corresponds to a spine of the same handlebody we have the following observation.

Lemma 9 Let $G$ be the fundamental group of a 3-manifold $M$ and $(g, h)$ and $\left(g^{\prime}, h^{\prime}\right)$ be Nielsen-equivalent generating pairs. Then $(g, h)$ is geometric if and only if $\left(g^{\prime}, h^{\prime}\right)$ is geometric.

\section{Compact 2-orbifolds with 2-generated fundamental group}

In this section we study generating pairs of Fuchsian groups, that is, of fundamental groups of 2-orbifolds. Throughout this article we assume that all 2-orbifolds have no reflection boundary lines. As all compact bad 2-orbifolds have finite fundamental groups it follows that any compact 2-orbifold is either Euclidean or hyperbolic or has 
finite fundamental group. We need to understand generating pairs of Euclidean and hyperbolic 2-orbifolds.

Lemma 10 Let $\mathcal{O}$ be a compact euclidean 2-orbifold with 2-generated fundamental group. Then one of the following holds:

(1) $\mathcal{O}=T^{2}, G=\pi_{1}(\mathcal{O})=\langle a, b \mid[a, b]\rangle$ and every pair of generators of $G$ is Nielsen equivalent to $(a, b)$.

(2) $\mathcal{O}=K B, G=\pi_{1}(\mathcal{O})=\left\langle a, b \mid a b a^{-1} b\right\rangle$ and every pair of generators of $G$ is Nielsen equivalent to $(a, b)$.

(3) $\mathcal{O}=S^{2}(p, q, r), 1=\frac{1}{p}+\frac{1}{q}+\frac{1}{r}$ and $G=\pi_{1}(\mathcal{O})=\left\langle s_{1}, s_{2}, s_{3} \mid s_{1}^{p}, s_{2}^{q}, s_{3}^{r}, s_{1} s_{2} s_{3}\right\rangle$ $=\left\langle s_{1}, s_{2} \mid s_{1}^{p}, s_{2}^{q},\left(s_{1} s_{2}\right)^{r}\right\rangle$. Moreover any generating pair $(g, h)$ of $G$ is Nielsenequivalent to $\left(s_{1}, s_{2}\right)$.

(4) $\mathcal{O}=P^{2}(2,2)$, that is, $G=\pi_{1}(\mathcal{O})=\left\langle x, s_{1}, s_{2} \mid s_{1}^{2}, s_{2}^{2}, x^{2} s_{1} s_{2}\right\rangle$. Moreover any generating pair is of $G$ is Nielsen-equivalent to $\left(x, s_{1}\right)$.

Proof Note first that every compact Euclidean 2-orbifold with 2-generated fundamental group occurs in this list as the only remaining compact Euclidean 2-orbifold $S^{2}(2,2,2,2)$ has a fundamental group of rank 3 . In the first two cases the assertion is obvious.

Suppose next that $\mathcal{O}=S^{2}(p, q, r)$ with $1=\frac{1}{p}+\frac{1}{q}+\frac{1}{r}$. This clearly implies that $(p, q, r)$ is $(3,3,3),(2,3,6)$ or $(2,4,4)$. Note first that any rotation $r$ that is a member of a generating pair of $G$ must generate a maximal cyclic subgroup as otherwise $G /\langle\langle r\rangle\rangle$ is non-cyclic. As any maximal finite cyclic subgroup is of order 2, 3, 4 or 6 this implies that any such rotation must be conjugate to $s_{i}^{ \pm 1}$ for some $i$. Let now $(g, h)$ be a generating pair. We can assume that $g$ is a rotation as two translations necessarily generate a subgroup of type $\mathbb{Z}^{2}$. Thus we can assume that $(g, h)=\left(s_{i}, h\right)$ for some $i$. Possibly after replacing $h$ with $h g$, we can assume that $h$ is also a rotation as otherwise $\langle g, h\rangle=\langle h, h g\rangle$ is free Abelian. It follows that $h$ is conjugate to $s_{j}$ for some $j$, again possible after replacing $h$ with $h^{-1}$.

Note first that if $g$ and $h$ are both of order 6 , that is, if $(p, q, r)=(2,3,6)$ and $g$ and $h$ are conjugate to $s_{3}$, then we can replace $g$ by $g h$ and obtain a rotation of order 3 . It is now obvious that $g$ and $h$ are the standard generating set of a Euclidean triangle group $\pi_{1}\left(S^{2}(l, m, n)\right)$ where $l$ is the order of $g, m$ is the order of $h$ and $n$ is the order of $g h$. If $g$ and $h$ generate $G$ then we must have $(l, m, n)$ is a permutation of $(p, q, r)$. The assertion follows easily.

We conclude by looking at the case $\mathcal{O}=P^{2}(2,2)$. Note that in this case $G$ splits as an amalgamated product over the cyclic subgroup generated by $x^{2}$, namely $G=$ 
$\langle x\rangle *\left\langle x^{2}=s_{2} s_{1}\right\rangle\left\langle s_{1}, s_{2}\right\rangle$. Now any generating pair of a 2 -generated amalgamated product is Nielsen equivalent to a generating pair $\left(g^{\prime}, h^{\prime}\right)$ such that a power of $g^{\prime}$ lies in the amalgam. Note first that $g^{\prime}$ cannot lie in the amalgam as $G /\left\langle\left\langle x^{2}\right\rangle\right\rangle$ is an infinite dihedral group and therefore not cyclic. As $\left\langle s_{2} s_{1}\right\rangle$ is isolated in $\left\langle s_{1}, s_{2}\right\rangle$ this implies that (possibly after conjugation) $g^{\prime} \in\langle x\rangle-\left\langle x^{2}\right\rangle$, that is, that $g^{\prime}=x^{2 l+1}$ for some $l \geq 0$. Suppose first that $l \geq 1$. After passing to the quotient

$$
G^{\prime}:=G /\left\langle\left\langle g^{\prime 2}\right\rangle\right\rangle=\left\langle x \mid x^{4 l+2}\right\rangle *\left\langle x^{2}=s_{2} s_{1}\right\rangle\left\langle s_{1}, s_{2} \mid s_{1}^{2}, s_{2}^{2},\left(s_{1} s_{2}\right)^{2 l+1}\right\rangle
$$

and replacing $g^{\prime}$ and $h^{\prime}$ with their images in $G^{\prime}$ we see that $g^{\prime}$ does not lie in any conjugate of the amalgam of the new amalgamated product. It therefore follows from Kapovich and Weidmann [12, Lemmas 2.1 and 2.2] that after replacing $h^{\prime}$ with $h^{\prime \prime}=g^{\prime z_{1}} h^{\prime} g^{\prime z_{2}}$ for some $z_{1}, z_{2} \in \mathbb{Z}$ a power of $h^{\prime \prime}$ also lies in $\langle x\rangle$. This however cannot be as $g^{\prime}$ and $h^{\prime \prime}$ both lie in the kernel of the quotient map $G^{\prime} \rightarrow G^{\prime} /\langle\langle x\rangle\rangle \cong \mathbb{Z}_{2}$, thus the original elements $g$ and $h$ could not have generated $G$. Thus we can assume that $g^{\prime}=x$. A simple normal form argument then shows that after Nielsen equivalence either $h^{\prime} \in\left\langle s_{1}, s_{2}\right\rangle$ or that $\left(g^{\prime}, h^{\prime}\right)$ do not generate $G$. The assertion now follows easily.

We lastly deal with hyperbolic 2-orbifolds. In the case of 2-orbifolds with orientable base space this situation has been completely discussed by Fine and Rosenberger [11], the remaining cases turn out be relatively easy.

Lemma 11 Let $\mathcal{O}$ be a compact hyperbolic 2-orbifold such that $G=\pi_{1}(\mathcal{O})$ is 2 -generated. Then one of the following holds:

(1) $\mathcal{O}=T^{2}(p), G=\left\langle a, b \mid[a, b]^{p}\right\rangle$ and every pair of generators of $G$ is Nielsen equivalent to $(a, b)$.

(2) $\mathcal{O}=K B(p), G=\left\langle a, b \mid\left(a b a^{-1} b\right)^{p}\right\rangle$ and every pair of generators of $G$ is Nielsen equivalent to $(a, b)$.

(3) $\mathcal{O}=P^{2}(p, q)$ with $p \geq 3$ and $q \geq 2$. Thus $G=\left\langle x, s_{1}, s_{2} \mid s_{1}^{p}, s_{2}^{q}, x^{2} s_{1} s_{2}\right\rangle$. Moreover any generating pair is Nielsen equivalent to $\left(x, s_{1}\right)$ unless $p=2$ and $q$ is odd in which case any generating set is Nielsen equivalent to either $\left(x, s_{1}\right)$ or $\left(x^{2}, x s_{1}\right)$.

(4) $\mathcal{O}=S^{2}(2,2,2,2 l+1), G=\left\langle s_{1}, \ldots, s_{4} \mid s_{1}^{2}, s_{2}^{2}, s_{3}^{2}, s_{4}^{2 l+1}, s_{1} s_{2} s_{3} s_{4}\right\rangle$ and any generating pair is Nielsen equivalent to $\left(s_{1} s_{2}, s_{1} s_{3}\right)$.

(5) $\mathcal{O}=S^{2}(p, q, r)$ with $1>\frac{1}{p}+\frac{1}{q}+\frac{1}{r}$ and $G=\left\langle s_{1}, s_{2} \mid s_{1}^{p}, s_{2}^{q},\left(s_{1} s_{2}\right)^{r}\right\rangle=$ $\left\langle s_{1}, s_{2}, s_{3} \mid s_{1}^{p}, s_{2}^{q}, s_{3}^{r}, s_{1} s_{2} s_{3}\right\rangle$. Moreover any generating pair $(g, h)$ is either Nielsen equivalent to a pair of type $\left(s_{i}^{n_{1}}, s_{j}^{n_{2}}\right)$ with $1 \leq i, j \leq 3$ and $n_{1}, n_{2} \in \mathbb{N}$ or one of the following holds: 
(a) $(p, q, r)=(2,3, r)$ with $\operatorname{gcd}(6, r)=1$ and $(g, h)$ is Nielsen equivalent to $\left(g^{\prime}, h^{\prime}\right)=\left(s_{1} s_{2} s_{1} s_{2}^{-1}, s_{2}^{-1} s_{1} s_{2} s_{1}\right)$.

(b) $(p, q, r)=(2,4, r)$ with odd $r$ and $(g, h)$ is Nielsen equivalent to $\left(g^{\prime}, h^{\prime}\right)=$ $\left(s_{1} s_{2}^{2}, s_{2}^{-1} s_{1} s_{2}^{-1}\right)$.

(c) $(p, q, r)=3,3, r$ with $\operatorname{gcd}(3, r)=1$ and $(g, h)$ is Nielsen equvalent to $\left(g^{\prime}, h^{\prime}\right)=\left(s_{1} s_{2}^{-1}, s_{2}^{-1} s_{1}\right)$.

(d) $(p, q, r)=(2,3,7)$ and $(g, h)$ is Nielsen equivalent to $\left(g^{\prime}, h^{\prime}\right)=$ $\left(s_{1} s_{2}^{-1} s_{1} s_{2} s_{1} s_{2}^{-1} s_{1} s_{2}^{-1} s_{1} s_{2}, s_{2}^{-1} s_{1} s_{2} s_{1} s_{2}^{-1} s_{1} s_{2} s_{1} s_{2} s_{1}\right)$.

Proof Note first that the list contains all compact hyperbolic 2-orbifolds with 2generated fundamental group by Zieschang [22] and Peczynski, Rosenberger and Zieschang [17]. The assertion of the lemma then follows from Fine and Rosenberger [11] except in situation (2) and (3).

If $\mathcal{O}=K B(p)$ then $G=\left\langle a, b, s \mid s^{p}, a b a^{-1}=s b^{-1}\right\rangle$, that is, $G$ is a HNN-extension of the group $\langle b, s\rangle \cong \mathbb{Z} * \mathbb{Z}_{p}$ with stable letter $a$ and associated subgroups $\langle b\rangle$ and $\left\langle s b^{-1}\right\rangle$. After Nielsen equivalence and conjugation we can assume that one generator, say $g$, lies in $\langle b\rangle$ (see, for example, Kapovich and Weidmann [12]) and it is not difficult to verify that $g$ cannot be a proper power of $b$ as $G /\left\langle\left\langle b^{n}\right\rangle\right\rangle$ is not cyclic for $n \geq 2$. Thus we can assume that $g=b$. It is further a simple calculation in normal forms to see that after replacing $h$ with $g^{z_{1}} h g^{z_{2}}$ for appropriate $z_{1}, z_{2} \in \mathbb{Z}$ we can assume that $h=a$ which proves the assertion, the essential step is to apply [5, Lemma 5].

Let $\mathcal{O}=P^{2}(p, q)$. As in the proof of Lemma 10 we see that we can assume that either $g=x$ or that $g=x^{2}$. If $g=x$ then we argue as in the proof of Lemma 10 and get $h=s_{1}$. If $g=x^{2}$ then it is easy to see that $h$ must be of type $x \bar{h}$ [5, Lemma 2] and that $h^{-1} g h=\bar{h}^{-1} x^{-1} x^{2} x \bar{h}=\bar{h}^{-1} x^{2} \bar{h}$ and $x^{2}$ must generate $\left\langle s_{1}, s_{2}\right\rangle$. The last assertion holds as if $\bar{h}^{-1} x^{2} \bar{h}$ and $x^{2}$ do not generated $\left\langle s_{1}, s_{2}\right\rangle$ then it is easily verified that they generate a free group in which case the original generators cannot have generated $G$ [5]. The assertion then follows from the fact that any orbifold of type $D(p, q)$ whose fundamental group is generated by two conjugates of the boundary curve is of type $D(2,2 l+1)$ (see Rost and Zieschang [18]).

Remark 12 In all cases of (5) in the above lemma we have that $\left[g^{\prime}, h^{\prime}\right]$ is a power of $s_{1} s_{2}$, namely $\left[g^{\prime}, h^{\prime}\right]=\left(s_{1} s_{2}\right)^{6}$ in case (a), $\left[g^{\prime}, h^{\prime}\right]=\left(s_{1} s_{2}\right)^{4}$ in case (b), $\left[g^{\prime}, h^{\prime}\right]=$ $\left(s_{1} s_{2}\right)^{3}$ in (c) and $\left[g^{\prime}, h^{\prime}\right]=\left(s_{1} s_{2}\right)^{4}$ in case (d). However the cases (a)-(c) are essentially different from case (d) in that in the first three cases the identity $\left[g^{\prime}, h^{\prime}\right]=\left(s_{1} s_{2}\right)^{r}$ holds in $\left\langle s_{1}, s_{2} \mid s_{1}^{p}, s_{2}^{q}\right\rangle$ while in case (d) the relation $\left(s_{1} s_{2}\right)^{r}$ is needed.

We conclude this section by studying the group $\pi_{1}\left(S^{2}(2,3,7)\right)$ in somewhat more detail. 
Lemma 13 Let $G=\pi_{1}\left(S^{2}(2,3,7)\right)=\left\langle s_{1}, s_{2} \mid s_{1}^{2}, s_{2}^{3},\left(s_{1} s_{2}\right)^{7}\right\rangle$. Then the pair $(g, h)$ given in 5 (d) above induces an inversion $\alpha$ of $G$. Moreover $\alpha$ is the inner automorphism given by

$$
w \mapsto\left(s_{2} s_{1} s_{2}^{-1}\right) w\left(s_{2} s_{1} s_{2}^{-1}\right)^{-1}
$$

Proof It clearly suffices to show that

$$
\left(s_{2} s_{1} s_{2}^{-1}\right) g\left(s_{2} s_{1} s_{2}^{-1}\right)^{-1}=g^{-1} \quad \text { and } \quad\left(s_{2} s_{1} s_{2}^{-1}\right) h\left(s_{2} s_{1} s_{2}^{-1}\right)^{-1}=h^{-1} .
$$

This is a simple computation.

\section{Seifert manifolds}

In this section we establish Theorem 5 for Seifert manifolds with 2-generated fundamental group except for those that fibre over the base space $P^{2}(p, q)$ or $S^{2}(2,2,2,2 l+1)$. Note first that any orientable Seifert manifold with spherical base space is either a lens space or can be fibered over a base space of type $S^{2}(p, q, r)$ with $\frac{1}{p}+\frac{1}{q}+\frac{1}{r}>1$. In the case of a lens space there is nothing to show and in the latter case any generating pair projects onto a pair of rotations in $\pi_{1}\left(S^{2}(p, q, r)\right) \leq S O(3)$. Together with the previous section this implies that for any orientable Seifert manifold $M$ with generating pair $(g, h)$ and base space different from $S^{2}(2,2,2,2 l+1)$ and $P^{2}(p, q)$ one of the following holds:

(1) After Nielsen equivalence we can assume that $g$ and $h$ map to elliptic elements of the base group.

(2) $M$ is Seifert fibered with base of type $T, T(p), K B$ or $K B(p)$.

(3) $M$ is fibered over a hyperbolic base space of type $S^{2}(p, q, r)$ with $(p, q, r)$ as in (5) of Lemma 11 and $(g, h)$ projects on the respective generating set of $\pi_{1}\left(S^{2}(p, q, r)\right)$.

We now deal with each of these three cases.

Lemma 14 Let $M$ be a Seifert manifold with 2-generated fundamental group. Any generating pair $(g, h)$ which projects to elliptic elements in the base group can be inverted by a hyperelliptic inversion of $\pi_{1}(M)$.

Proof The assumption that the images $\pi(g)$ and $\pi(h)$ of the two generators are elliptic implies that $M$ is Seifert fibered over a base of type $S^{2}(p, q, r)$. After possibly replacing $g$ and $h$ with roots we may assume that the element $f$ corresponding to the fibre is a power of $g$ and $h$. Then $g$ and $h$ are inverted by the inversion induced 
by the Montesinos involution $\tau$ on $M$ (see Montesinos $[15 ; 16]$ ). This involution is geometric, reverses the orientation of the base $S^{2}(p, q, r)$ while fixing each singular point and reverses the orientation of the generic fiber $f$. The quotient map $M \rightarrow M / \tau$ is a 2-fold covering of $S^{3}$ branched over a Montesinos link $L$ with 3 bridges by Boileau and Zieschang [7]. Therefore $\tau$ is induced by the hyperelliptic involution of a genus 2 Heegaard splitting of $M$ obtained by lifting the 3-bridge presentation of $L$.

Lemma 15 Let $M$ be a Seifert manifold with 2-generated fundamental group that fibers over $T^{2}, T^{2}(p), K B$ or $K B(p)$. Then any generating pair of $\pi_{1}(M)$ is geometric. In particular it can be inverted by a hyperelliptic inversion.

Proof We consider $T^{2}$ as $T^{2}(1)$ and $K B$ as $K B(1)$. Recall that the fundamental group is either of the form $\left.\langle a, b, f|[a, f], b], f],[a, b]^{p}=f^{e}\right\rangle$ or $\langle a, b, f| a f a^{-1}=$ $\left.f^{-1}, b f b^{-1}=f^{-1},\left(a^{2} b^{2}\right)^{p}=f^{e}\right\rangle$ with $e=1$ as otherwise $\pi_{1}(M)$ is not 2generated (see Boileau and Zieschang [6]).

In both cases it is easy to see that $(a, b)$ is a geometric generating set corresponding to a horizontal Heegaard splitting. It is futher clear that any pair $\left(a f^{l}, b f^{k}\right)$ is also a generating set as $\left[a f^{l}, b f^{k}\right]=[a, b]$ and $\left(a f^{l}\right)^{2}\left(b f^{l}\right)^{2}=a^{2} b^{2}$. The map $a \mapsto a f^{l}$ and $b \mapsto b f^{k}$ clearly extends to an isomorphism which is induced by homeomorphism. Thus $\left(g f^{l}, b f^{k}\right)$ is also geometric.

We will (sometimes implicitly) use the following simple observation.

Lemma 16 Let $M$ be a Seifert manifold fibered over $S^{2}(p, q, r)$ and

$$
\pi: \pi_{1}(M) \rightarrow\left\langle s_{1}, s_{2} \mid s_{1}^{p}, s_{2}^{q},\left(s_{1} s_{2}\right)^{r}\right\rangle
$$

be the projection. Let $U=\left\langle g_{1}, g_{2}, x_{1}, \ldots, x_{k}\right\rangle \leq \pi_{1}(M)$ such that $\pi\left(g_{i}\right)=s_{i}$ for $i=$ 1,2 and $x_{i} \in\langle f\rangle=\operatorname{ker} \pi$ for $1 \leq i \leq k$. Then $U \cap\langle f\rangle=\left\langle x_{1}, \ldots, x_{k}, g_{1}^{p}, g_{2}^{q},\left(g_{1} g_{2}\right)^{r}\right\rangle$.

Proposition 17 Let $M$ be a Seifert manifold with hyperbolic base space $S^{2}(2,3, r)$ where $\operatorname{gcd}(6, r)=1, S^{2}(2,4, r)$ with odd $r$ or $S^{2}(3,3, r)$ with $\operatorname{gcd}(r, 3)=1$.

Let $(g, h)$ be a generating pair of $\pi_{1}(M)$ that projects onto the generating pair of the base group spelt out in Lemma 11 (5) (a)-(c). Then the following hold:

(1) If $(g, h)$ is invertible then $(g, h)$ is geometric and corresponds to a horizontal Heegaard splitting of genus 2 .

(2) There exist non-invertible generating pairs of this type. 
Proof We give all details in the case of $S^{2}(2,3, r)$ with $\operatorname{gcd}(6, r)=1$ and then comment on the other cases.

Note that $r=6 p+\varepsilon$ with $\varepsilon \in\{-1,1\}$ and $p \geq 1$. Thus we assume that $g=$ $s_{1} s_{2} s_{1} s_{2}^{-1} f^{k}$ and $h=s_{2}^{-1} s_{1} s_{2} s_{1} f^{l}$ generate the group

$$
\begin{aligned}
G & =\left\langle s_{1}, s_{2}, s_{3}, f \mid s_{1}^{2}=f, s_{2}^{3}=f, s_{3}^{r}=f^{n}, s_{1} s_{2} s_{3}=f^{m}\right\rangle \\
& =\left\langle s_{1}, s_{2}, f \mid s_{1}^{2}=f, s_{2}^{3}=f,\left(s_{1} s_{2}\right)^{r}=f^{q}\right\rangle
\end{aligned}
$$

where $q=r m-n$.

Suppose now that $(g, h)$ is invertible and let $\alpha$ be the induced inversion. The homeomorphism corresponding to $\alpha$ must be isotopic to the identity as $\alpha$ preserves the fibre. Thus $\alpha$ must be an inner automorphism. It is easily verified that $g$ is conjugate to $g^{-1}$ and $h$ is conjugate to $h^{-1}$ if and only if $k=l=-1$.

We need some calculations. Note first that

$$
\begin{aligned}
{[g, h]=s_{1} s_{2} s_{1} s_{2}^{-1} \cdot s_{2}^{-1} s_{1} s_{2} s_{1} } & \cdot s_{2} s_{1}^{-1} s_{2}^{-1} s_{1}^{-1} \cdot s_{1}^{-1} s_{2}^{-1} s_{1}^{-1} s_{2} \\
& =s_{1} s_{2} s_{1} s_{2}^{-2} s_{1} s_{2} s_{1} s_{2} s_{1}^{-1} s_{2}^{-2} s_{1}^{-1} s_{2} f^{-1}=\left(s_{1} s_{2}\right)^{6} f^{-5}
\end{aligned}
$$

and therefore

$$
\begin{aligned}
{[g, h]^{r} } & =\left(s_{1} s_{2}\right)^{6 r} f^{-5 r}=f^{6 q-5 r} \\
\text { and } \quad c & :=[g, h]^{p}=\left(s_{1} s_{2}\right)^{6 p} f^{-5 p}=\left(s_{1} s_{2}\right)^{r-\varepsilon} f^{-5 p}=\left(s_{1} s_{2}\right)^{-\varepsilon} f^{q-5 p} .
\end{aligned}
$$

We define $g^{\prime}:=c^{2 \varepsilon} g$, that is, we have

$$
\begin{aligned}
g^{\prime} & =\left(s_{1} s_{2}\right)^{-2} s_{1} s_{2} s_{1} s_{2}^{-1} f^{k+2 \varepsilon q-10 \varepsilon p} \\
& =s_{2}^{-2} f^{k+2 \varepsilon q-10 \varepsilon p}=s_{2} f^{k-1+2 \varepsilon q-10 \varepsilon p} \\
\text { and } \quad g^{\prime 3} & =f \cdot f^{3 k-3+6 \varepsilon q-30 \varepsilon p}=f^{3 k-2+6 \varepsilon q-30 \varepsilon p}=f^{3 k+3+6 \varepsilon q-5 \varepsilon r} .
\end{aligned}
$$

We then put $h^{\prime}:=g^{\prime-1} c^{-\varepsilon} h$, that is,

$$
\begin{aligned}
h^{\prime} & =s_{2}^{-1} f^{-k+1-2 \varepsilon q+10 \varepsilon p}\left(s_{1} s_{2}\right) f^{-\varepsilon q+\varepsilon 5 p} s_{2}^{-1} s_{1} s_{2} s_{1} f^{l} \\
& =s_{1} f^{-k+l+2-3 \varepsilon q+\varepsilon 15 p}
\end{aligned}
$$

and

$$
h^{\prime 2}=f^{-2 k+2 l+5-6 \varepsilon q+\varepsilon 30 p}=f^{-2 k+2 l-6 \varepsilon q+5 \varepsilon r} .
$$

In the case $l=k=-1$ a simple calculation further shows that $h^{\prime} g^{\prime}=c^{-\varepsilon}$ and it follows that $\langle g, h\rangle=\langle c, g, h\rangle=\left\langle g^{\prime}, h^{\prime}\right\rangle$. Lemma 16 then implies that $\langle g, h\rangle \cap\langle f\rangle=$ $\left\langle g^{\prime 3}, h^{\prime 2},\left(h^{\prime} g^{\prime}\right)^{r}\right\rangle=\left\langle f^{6 q-5 r}\right\rangle$. As we assume that $(g, h)$ is a generating set this implies that $6 q-5 r \in\{-1,1\}$, in particular $[g, h]$ is the $r^{\text {th }}$ root of the fibre, thus there 
is a horizontal splitting with respect to which $(g, h)$ is a geometric generating pair. It further follows from the above computations that for many other choices of $l$ and $k$ the pair $(g, h)$ is also a generating pair, which must be non-invertible.

The case of a base space of type $S^{2}(2,4, r)$ and odd $r$ is completely analogous, again the induced involution must be isotopic to the identity.

In the case of a base space of type $S^{2}(3,3, r)$ and $r=3 p+\varepsilon$ with $\varepsilon \in\{-1,1\}$ the argument is different. We assume that there is an invertible generating pair $(g, h)$ of

$$
\begin{aligned}
G & =\left\langle s_{1}, s_{2}, s_{3}, f \mid s_{1}^{3}=f, s_{2}^{3}=f^{\eta}, s_{3}^{r}=f^{n}, s_{1} s_{2} s_{3}=f^{m}\right\rangle \\
& =\left\langle s_{1}, s_{2}, f \mid s_{1}^{3}=f, s_{2}^{3}=f^{\eta},\left(s_{1} s_{2}\right)^{r}=f^{q}\right\rangle
\end{aligned}
$$

where $\eta \in\{-1,1\}$ and $q=r m-n$ such that $(g, h)=\left(s_{1} s_{2}^{-1} f^{k}, s_{2}^{-1} s_{1} f^{l}\right)$.

As before we see that a power of the commutator $[g, h]$ is a power of $f$. This implies that the induced inversion $\alpha$ of $G$ must fix $f$. Thus it is induced by a fiber preserving involution of the manifold that preserves the orientation by Zieschang and Zimmerman [24], see also Zieschang [23]. This involution cannot be isotopic to the identity as $g=s_{1} s_{2}^{-1} f^{k}$ is not conjugate to $g^{-1}=s_{2} s_{1}^{-1} f^{-k}$ for any choice of $k$. This can be seen as $s_{1} s_{2}^{-1}$ and $s_{2} s_{1}^{-1}$ are not conjugate in the base group.

It follows that the involution exchanges the two exceptional fibres with invariants $(3,1)$ and $(3, \eta)$, in particular $\eta=1$. Thus the inversion $\alpha$ must map $s_{1}$ to $s_{2}$ and $s_{2}$ to $s_{1}$ which implies that $k=l=0$. The remainder of the argument is as before.

For Seifert manifolds $M$ that fibre over the base space $S^{2}(2,3,7)$, we will now study generating pairs that project onto the generating pair of the base described in Lemma 11 (5) (d). Recall that we have

$$
\begin{aligned}
\pi_{1}(M) & =\left\langle s_{1}, s_{2}, s_{3}, f \mid s_{1}^{2}=f, s_{2}^{3}=f, s_{3}^{7}=f^{n}, s_{1} s_{2} s_{3}=f^{m}\right\rangle \\
& =\left\langle s_{1}, s_{2}, f \mid s_{1}^{2}=f, s_{2}^{3}=f,\left(s_{1} s_{2}\right)^{7}=f^{q}\right\rangle
\end{aligned}
$$

with $q:=7 m-n$. This shows in particular that there is a 1-parameter family of Seifert manifolds with base space $S^{2}(2,3,7)$. The following lemma implies that there are plenty of generating pairs of $\pi_{1}(M)$ that project onto the generating set of the base group described in Lemma 11 5(d).

Lemma 18 Let $M$ be an orientable Seifert manifold with base space $S^{2}(2,3,7)$ and $G=\pi_{1}(M)=\left\langle s_{1}, s_{2}, f \mid s_{1}^{2}=f, s_{2}^{3}=f,\left(s_{1} s_{2}\right)^{7}=f^{q}\right\rangle$ with $q$ as above.

Then $G$ is generated by two elements

$$
g=s_{1} s_{2}^{-1} s_{1} s_{2} s_{1} s_{2}^{-1} s_{1} s_{2}^{-1} s_{1} s_{2} f^{k} \quad \text { and } \quad h=s_{2}^{-1} s_{1} s_{2} s_{1} s_{2}^{-1} s_{1} s_{2} s_{1} s_{2} s_{1} f^{l}
$$


iff $\langle f\rangle$ is generated by

$$
f^{k+l+5}, \quad f^{2 k-20 q+121}, \quad f^{6 k+6 l+12 q-40} \quad \text { and } f^{-7 k-14 l-97 q+511} .
$$

Proof Note first that

$$
\begin{aligned}
g h & =s_{1} s_{2}^{-1} s_{1} s_{2} s_{1} s_{2}^{-1} s_{1} s_{2}^{-1} s_{1} s_{2} f^{k} s_{2}^{-1} s_{1} s_{2} s_{1} s_{2}^{-1} s_{1} s_{2} s_{1} s_{2} s_{1} f^{l} \\
& =s_{1} s_{2}^{-1} s_{1} s_{2} s_{1} s_{2}^{-1} s_{1} s_{2}^{-1} s_{1}^{2} s_{2} s_{1} s_{2}^{-1} s_{1} s_{2} s_{1} s_{2} s_{1} f^{k+l} \\
& =s_{1} s_{2}^{-1} s_{1} s_{2} s_{1} s_{2}^{-1} s_{1}^{2} s_{2}^{-1} s_{1} s_{2} s_{1} s_{2} s_{1} f^{k+l+1} \\
& =s_{1} s_{2}^{-1} s_{1} s_{2} s_{1} s_{2}^{-2} s_{1} s_{2} s_{1} s_{2} s_{1} f^{k+l+2} \\
& =s_{1} s_{2}^{-1}\left(s_{1} s_{2} s_{1} s_{2} s_{1} s_{2} s_{1} s_{2} s_{1}\right) f^{k+l+1} \\
& =s_{1} s_{2}^{-1} s_{2}^{-1} s_{1}^{-1} s_{2}^{-1} s_{1}^{-1} s_{2}^{-1} f^{q+k+l+1} \\
& =s_{1} s_{2}^{-2} s_{1}^{-1} s_{2}^{-1} s_{1}^{-1} s_{2}^{-1} f^{q+k+l+1} \\
& =s_{1} s_{2} s_{1} s_{2}^{-1} s_{1}^{-1} s_{2}^{-1} f^{q+k+l-1}
\end{aligned}
$$

Similar arguments show that

$$
g^{-1} h^{-1}=s_{2} s_{1} s_{2}^{-1} s_{1} s_{2} s_{1} s_{2} f^{q-k-l-13}
$$

and therefore

$$
\begin{aligned}
& {[g, h]=g h g^{-1} h^{-1}=\left(s_{1} s_{2}\right)^{4} f^{2 q-15}} \\
& \text { and } \quad c:=[g, h]^{2}=s_{1} s_{2} f^{5 q-30} \text {. }
\end{aligned}
$$

Clearly $\langle g, h\rangle=\langle g, h, c\rangle$ and therefore $\langle g, h\rangle=\left\langle g^{\prime}:=c^{-1} g c^{-1}, h^{\prime}:=c h c, c\right\rangle$ where

$$
\text { and } \quad \begin{aligned}
g^{\prime} & =f^{-5 q+30} s_{2}^{-1} s_{1}^{-1}\left(s_{1} s_{2}^{-1} s_{1} s_{2} s_{1} s_{2}^{-1} s_{1} s_{2}^{-1} s_{1} s_{2} f^{k}\right) f^{-5 q+30} s_{2}^{-1} s_{1}^{-1} \\
& =s_{2} s_{1} s_{2} s_{1} s_{2}^{-1} s_{1}^{-1} s_{2}^{-1} f^{-10 q+k+60} \\
& =s_{1} s_{2} f^{5 q-30}\left(s_{2}^{-1} s_{1} s_{2} s_{1} s_{2}^{-1} s_{1} s_{2} s_{1} s_{2} s_{1} f^{l}\right) s_{1} s_{2} f^{5 q-30} \\
& =s_{2} s_{1} s_{2}^{-1} s_{1} s_{2} s_{1}^{-1} s_{2}^{-1} f^{10 q+l-56} .
\end{aligned}
$$

Conjugation with $s_{2} s_{1}$ shows that $\langle g, h\rangle$ is conjugate to $\left\langle g^{\prime \prime}, h^{\prime \prime}, c^{\prime}\right\rangle$ with

$$
\begin{aligned}
g^{\prime \prime} & =s_{2} s_{1} s_{2}^{-1} f^{-10 q+k+60}, \\
h^{\prime \prime} & =s_{2}^{-1} s_{1} s_{2} f^{10 q+l-56} \\
\text { and } \quad c^{\prime} & =s_{1}^{-1} s_{2}^{-1}\left(s_{1} s_{2} f^{5 q-30}\right) s_{2} s_{1}=s_{1} s_{2}^{-1} s_{1} s_{2}^{-1} s_{1} f^{5 q-30} .
\end{aligned}
$$


We further replace $c^{\prime}$ with $c^{\prime \prime}=g^{\prime \prime} c^{\prime} h^{\prime \prime}$ which gives

$$
\begin{aligned}
c^{\prime \prime} & =s_{2} s_{1} s_{2}^{-1} f^{-10 q+k+60}\left(s_{1} s_{2}^{-1} s_{1} s_{2}^{-1} s_{1} f^{5 q-30}\right) s_{2}^{-1} s_{1} s_{2} f^{10 q+l-56} \\
& =s_{2}\left(s_{1}^{-1} s_{2}^{-1} s_{1}^{-1} s_{2}^{-1} s_{1}^{-1} s_{2}^{-1} s_{1}^{-1} s_{2}^{-1} s_{1}^{-1}\right) s_{2} f^{5 q+k+l-21} \\
& =s_{2}^{-1} s_{1} s_{2} s_{1} s_{2}^{-1} f^{4 q+k+l-19}
\end{aligned}
$$

and go on by replacing $c^{\prime \prime}$ with $c^{\prime \prime \prime}=h^{\prime \prime} c^{\prime \prime} g^{\prime \prime}$, that is,

$$
c^{\prime \prime \prime}=s_{2}^{-1} f^{4 q+2 k+2 l-13} \text {. }
$$

We further replace $g^{\prime \prime}$ and $h^{\prime \prime}$ by

$$
\begin{aligned}
& g^{\prime \prime \prime}=c^{\prime \prime \prime} g^{\prime \prime} c^{\prime \prime \prime-1}=s_{1} f^{-10 q+k+60} \\
& \text { and } \quad h^{\prime \prime \prime}=c^{\prime \prime \prime-1} h^{\prime \prime} c^{\prime \prime \prime}=s_{1} f^{10 q+l-56} \text {. }
\end{aligned}
$$

We lastly replace $h^{\prime \prime \prime}$ with $\bar{h}=g^{\prime \prime \prime} h^{\prime \prime \prime}=f^{k+l+5}$. These changes clearly preserve the subgroup, thus $\langle g, h\rangle=\left\langle g^{\prime \prime \prime}, \bar{h}, c^{\prime \prime \prime}\right\rangle$. Lemma 16 now implies that

$$
\langle f\rangle \cap\langle g, h\rangle=\left\langle\bar{h},\left(g^{\prime \prime \prime}\right)^{2},\left(c^{\prime \prime \prime}\right)^{3},\left(g^{\prime \prime \prime}\left(c^{\prime \prime \prime}\right)^{-1}\right)^{7}\right\rangle
$$

where

$$
\begin{aligned}
\left(g^{\prime \prime \prime}\right)^{2} & =f^{-20 q+2 k+121}, \\
\left(c^{\prime \prime \prime}\right)^{3} & =f^{12 q+6 k+6 l-40} \\
\text { and } \quad\left(g^{\prime \prime \prime}\left(c^{\prime \prime \prime}\right)^{-1}\right)^{7} & =f^{-97 q-7 k-14 l+511 .} .
\end{aligned}
$$

This clearly implies the assertion of the lemma.

Invertible generating pairs of this type however turn out to be extremely rare, in fact there is only one manifold fibered over $S^{2}(2,3,7)$ that admits one, and for this manifold the invertible generating pair is unique.

Lemma 19 Let $M$ be as above and

$$
(g, h)=\left(s_{1} s_{2}^{-1} s_{1} s_{2} s_{1} s_{2}^{-1} s_{1} s_{2}^{-1} s_{1} s_{2} f^{k}, s_{2}^{-1} s_{1} s_{2} s_{1} s_{2}^{-1} s_{1} s_{2} s_{1} s_{2} s_{1} f^{l}\right) .
$$

Then $(g, h)$ is an invertible generating pair if and only if $q=6, k=-2$ and $l=-3$, that is, $M$ is the Brieskorn homology sphere

$$
\Sigma(2,3,7)=\left\{z_{1}^{2}+z_{2}^{3}+z_{3}^{7}=0\right\} \cap\left\{\left|z_{1}\right|^{2}+\left|z_{2}\right|^{2}+\left|z_{3}\right|^{2}=1\right\} .
$$

Moreover this generating set is not geometric. 
Proof Suppose first that $(g, h)$ is invertible. Let $\alpha$ be the induced automorphism of $G$. As the fibre is a power of $[g, h]$ and $\alpha([g, h])=\left[g^{-1}, h^{-1}\right]$ is conjugate to $[g, h]$ it follows that $\alpha$ preserves $f$. Thus $\alpha$ is induced by a homeomorphism that is isotopic to the identiy, that is, $\alpha$ is an inner automorphism. Note that the inner automorphism must be conjugation with $s_{2} s_{1} s_{2}^{-1} f^{l}$ for some $l$ by Lemma 13 as $\alpha$ induces an involution on the base group. As the value of $l$ has no effect in the conjugation we can assume that $l=0$. Note that $\left(s_{2} s_{1} s_{2}^{-1}\right)^{-1}=s_{2} s_{1}^{-1} s_{2}^{-1}=f^{-1} s_{2} s_{1} s_{2}^{-1}$. We obtain

$$
\begin{aligned}
f^{-1} s_{2} s_{1} s_{2}^{-1} \cdot g \cdot s_{2} s_{1} s_{2}^{-1} & =f^{-1} s_{2} s_{1} s_{2}^{-1} \cdot s_{1} s_{2}^{-1} s_{1} s_{2} s_{1} s_{2}^{-1} s_{1} s_{2}^{-1} s_{1} s_{2} f^{k} \cdot s_{2} s_{1} s_{2}^{-1} \\
& =s_{2} s_{1} s_{2}^{-1} s_{1} s_{2}^{-1} s_{1} s_{2}\left(s_{1}^{-1} s_{2}^{-1} s_{1}^{-1} s_{2}^{-1} s_{1}^{-1} s_{2}^{-1} s_{1}^{-1} s_{2}^{-1}\right) f^{k+4} \\
& =s_{2} s_{1} s_{2}^{-1} s_{1} s_{2}^{-1} s_{1} s_{2} s_{2} s_{1} s_{2} s_{1} s_{2} s_{1} f^{-q+k+4} \\
& =s_{2}\left(s_{1}^{-1} s_{2}^{-1} s_{1}^{-1} s_{2}^{-1} s_{1}^{-1} s_{2}^{-1} s_{1}^{-1}\right) s_{2} s_{1} s_{2} s_{1} f^{-q+k+9} \\
& =s_{2}\left(s_{2} s_{1} s_{2} s_{1} s_{2} s_{1} s_{2}\right) s_{2} s_{1} s_{2} s_{1} f^{-2 q+k+9} \\
& =s_{2}^{-1} s_{1}^{-1} s_{2} s_{1}^{-1} s_{2} s_{1}^{-1} s_{2}^{-1} s_{1}^{-1} s_{2} s_{1}^{-1} f^{-2 q+k+16}
\end{aligned}
$$

It follows that $f^{-1} s_{2} s_{1} s_{2}^{-1} \cdot g \cdot s_{2} s_{1} s_{2}^{-1}=g^{-1}$ if and only if $-k=-2 q+k+16$, that is, if $k=q-8$. The analogous calculation for $h$ shows that $f^{-1} s_{2} s_{1} s_{2}^{-1} \cdot h \cdot s_{2} s_{1} s_{2}^{-1}=h^{-1}$ if and only if $-l=-2 q+l-6$, that is, if $l=-q+3$.

Lemma 18 implies that $\langle g, h\rangle \cap\langle f\rangle$ is generated by

$$
\begin{aligned}
f^{k+l+5} & =f^{0}, \\
f^{2 k-20 q+121} & =f^{2(q-8)-20 q+121} \\
& =f^{-18 q+105}, \\
f^{6 k+6 l+12 q-40} & =f^{6(q-8)+6(-q+3)+12 q-40} \\
& =f^{12 q-70} \\
\text { and } \quad f^{-7 k-14 l-97 q+511} & =f^{-7(q-8)-14(-q+3)-97 q+511} \\
& =f^{-90 q+525} .
\end{aligned}
$$

Thus $\langle g, h\rangle \cap\langle f\rangle=\left\langle f^{6 q-35}\right\rangle$. Thus $\langle g, h\rangle \cap\langle f\rangle=\langle f\rangle$ and therefore $G=\langle g, h\rangle$ iff $q=6$ and therefore also $k=-2$ and $l=-3$.

To see that this generating set if not geometric it suffices to recall that $M$ only admits two distinct Heegaard splitting, one vertical and one horizontal corresponding to the generating pair discussed in Proposition 17. The generating pairs corresponding to these splittings are not Nielsen equivalent to $(g, h)$ by Lemma 11. This implies that 
$(g, h)$ is not geometric. The generating pair corresponding to the horizontal splitting however happens to induce the same involution of the manifold.

\section{Inversion on aspherical graph manifolds}

In this section $M$ will be a closed, orientable, aspherical, sufficiently complicated graph manifold, that is, it either admits a non-empty splitting along a finite collection of essential and pairwise non parallel tori into Seifert fibered pieces or it is Seifert fibered with a hyperbolic base. Such a decomposition is called a JSJ-decomposition. We show that any inversion of $\pi_{1}(M)$ can be realized by an involution which preserves the JSJ-splitting and the Seifert structures of the pieces. More precisely:

Proposition 20 Let $M$ be a closed, orientable, aspherical, sufficiently complicated graph manifold. Then any inversion $\alpha$ of $\pi_{1}(M)$ can be realized by an involution $\tau$ on $M$ with the following properties.

(1) The involution $\tau$ respects the JSJ-decomposition of $M$ and the Seifert fibered structures on the JSJ-pieces.

(2) The underlying space of the quotient orbifold $M / \tau$ is homeomorphic to $S^{3}$ and the map $p: M \rightarrow|M / \tau|=S^{3}$ is a 2 -fold covering branched along a prime and unsplittable link $L$ with at most 3-components. Moreover if $L$ has $\geq 2$ components either $L=K_{1} \cup K_{2}$ where $K_{1}$ is unknotted and $K_{2}$ is a 2-bridge knot, or $L=K_{1} \cup K_{2} \cup K_{3}$ where each component $K_{i}$ is unknotted and each sublink $K_{i} \cup K_{j}, i \neq j \in\{1,2,3\}$ is a 2 -bridge link.

(3) The inversion $\alpha$ determines the pair $\left(S^{3}, L\right)$ up to homeomorphism and is hyperelliptic if and only if $L$ is a 3-bridge link.

Proof An inversion $\alpha \in \operatorname{Aut}\left(\pi_{1}(M)\right)$ of the generating pair $(g, h)$ gives an extension of $\pi_{1}(M)$ :

$$
1 \rightarrow \pi_{1}(M) \rightarrow E \rightarrow \mathbb{Z} / 2 \mathbb{Z} \rightarrow 1,
$$

such that $E=\left\langle\pi_{1}(M), t \mid t^{2}, t \gamma t^{-1}=\alpha(\gamma), \forall \gamma \in \pi_{1}(M)\right\rangle$.

The key step of the proof is to realize $E$ as a group of orientation preserving homeomorphisms of the universal cover $\widetilde{M}$ of $M$ in such a way that the subgroup $\pi_{1}(M)$ is the deck transformation group of the cover $\widetilde{M} \rightarrow M$.

In the Seifert fibered case, using Kerckhoff's proof of the Nielsen's conjecture [13], one can even realize $E$ as a group of fiber preserving homeomorphisms of the universal cover $\widetilde{M}$ of $M$, according to Zimmerman and Zieschang [25; 24], see also [23]. 
In the case of a proper graph manifold this realization of $E$ as group of homeomorphisms of the universal cover $\widetilde{M}$ is achieved by Zimmerman [26, Section D].

Now it is clear that the quotient group $E / \pi_{1}(M) \cong \mathbb{Z} / 2 \mathbb{Z}$ acts on $M$ and is generated by the projection $\tau$ on $M$ of the involution $t$ on $\widetilde{M}$. Since $\widetilde{M}$ is homeomorphic to $\mathbb{R}^{3}, t$ must have non-empty fixed point set, hence $\tau$ has non-empty fixed point set.

The group $E$ is the orbifold fundamental group of the quotient orbifold $\widetilde{M} / E=M / \tau$. It is generated by the three involutions $t g, t h, t$, hence the fundamental group of the underlying space $|M / \tau|$ is trivial (see Armstrong [2] and Thurston [20, Chapter 13]). Since the orbifold $M / \tau$ is either Seifert fibered or splits along a finite collection of disjoint euclidean 2-suborbifolds into Seifert fibered pieces, it follows that the underlying space $|M / \tau|$ must be homeomorphic to the sphere $S^{3}$. Therefore $p: M \rightarrow$ $|M / \tau|=S^{3}$ is a 2 -fold covering branched along a link $L$ with at most 3 components since $\operatorname{dim} H_{1}(M, \mathbb{Z} / 2 \mathbb{Z}) \leq 2$ because $\pi_{1}(M)$ is 2 -generated. Moreover $L$ is prime and unsplittable since $M$ is irreducible.

The group $E$ is the so called $\pi$-orbifold group of the link $L$ (see Boileau and Zimmerman [8]). It can be computed from the fundamental group $\pi_{1}\left(S^{3} \backslash L\right)$ of the link $L$ by killing the squares of the meridian elements $\mu_{i}$ :

$$
E=\pi_{1}\left(S^{3} \backslash L\right) /\left\langle\left\langle\mu_{i}^{2}\right\rangle\right\rangle .
$$

The proof of the Smith conjecture shows that a knot is unknotted if and only if its $\pi$-orbifold group is $\mathbb{Z} / 2 \mathbb{Z}$. Moreover a prime and unsplittable link is a 2 -bridge link if and only if its $\pi$-orbifold group is dihedral (see [8]). The $\pi$-orbifold group of a component $K$ of $L$ can be obtained from the $\pi$-orbifold group $E$ of $L$ by killing the meridians of the other components of $L$. Then the assertion on the components of $L$ when $L$ has at least 2 components, follows readily from the two previous facts.

By Zimmerman [27, Theorems 0.1 and 1.1], the inversion $\alpha$ determines the involution $\tau$ up to conjugation by a homeomorphism homotopic to the identity, hence $\alpha$ determines the link L. Therefore $\alpha$ is hyperelliptic if and only if $\tau$ is induced by the hyperelliptic involution of a Heegaard splitting of genus 2. By Birman and Hilden [3] this is equivalent to saying that the link $L$ has 3 bridges.

The proof of Theorem 5 follows from the results of Section 4 and Corollary 21 below.

Corollary 21 Let $M$ be an orientable Seifert manifold with base orbifold $P^{2}(p, q)$ or $S^{2}(2,2,2,2 l+1)$. Then any inversion of the fundamental group of $M$ is hyperelliptic. 
Proof Let $\alpha$ be an inversion of $\pi_{1}(M)$. By Proposition 20 it is induced by a fiber preserving involution $\tau$. Let $p: M \rightarrow|M / \tau|=S^{3}$ be the associated 2-fold covering of $S^{3}$ branched along a link $L$.

If the base space is $S^{2}(2,2,2,2 l+1)$, we apply the argument of Boileau and Zieschang [6, Lemma 3.3]. The link $L$ has 3 components since $\operatorname{dim} H_{1}(M, \mathbb{Z} / 2 \mathbb{Z})=2$. We distinguish two cases depending on the action of $\tau$ on the fibres:

Case 1: $\tau$ reverses the orientation of the fibres. Since the quotient space $|M / \tau|=$ $S^{3} \tau$ is a Montesinos involution (see [15] and [16, Chapter 4]). Thus $L$ is a Montesinos link with 3 components, one of which is a knotted 2-bridge knot (see case (a) of [6, Lemma 3.3]). Hence $\tau$ cannot induce an inversion.

Case 2: $\tau$ preserves the orientation of the fibres. Then Case 3.5 of the proof of $[6$, Lemma 3.3] shows that the quotient space $|M / \tau|=S^{3}$ and $L$ has 3 components, if and only if $L$ is the 3-bridge torus link $T(3,3(2 l+1))$. Hence $\tau$ induces an inversion of $\pi_{1}(M)$ if and only if it is hyperelliptic.

When the base space is $P^{2}(p, q)$ then $\tau$ must reverse the orientation of the singular fibres since the quotient space $|M / \tau|=S^{3}$. Hence $\tau$ is a Montesinos involution and $L$ is a generalized Montesinos link (see [15] and [16, Chapter 4]). It follows from Boileau and Zieschang [7, Theorem 2.1] that the bridge number of $L$ is 3. Therefore $\alpha$ is a hyperelliptic inversion.

Remark 22 The same type of arguments can be applied more generally to show that an inversion of the fundamental group of a sufficiently complicated closed orientable Seifert manifolds is hyperelliptic. The proof would follow from the following two facts:

(1) The bridge number of a (generalized) Montesinos link $L$ with 2 -fold branched cover $M$ is rank $\pi_{1}(M)+1$, except in the case when $M$ has base space $S^{2}(2,2, \ldots, 2,2 l+1)$ with an even number of singular points, see [7].

(2) The bridge number of a torus link whose $\pi$-orbifold group is generated by $k$ elements of order 2 is at most $k$.

However the interest of the algebraic proof given in the previous Section 4 is to give more precise results: it shows that many Heegaard genus 2 Seifert manifolds have non invertible generating pairs and even that the Brieskorn homology sphere admits an invertible generating pair which is not geometric. 


\section{Graph manifolds}

Theorem 5 clearly implies Theorem 3 for Seifert manifolds. In this section we consider graph-manifolds which are not Seifert fibered, that is, graph-manifolds that have a non-trivial JSJ-decomposition.

The purpose of the section is to establish the following proposition, which finishes the proof of Theorem 3 together with Theorem 5.

Proposition 23 The fundamental group of a closed, orientable graph manifold of Heegaard genus greater than 2 does not admit an inversion.

We need only to consider the case where the graph manifold is not Seifert fibered. The following lemma precisely describes such graph manifolds of Heegaard genus 3 .

Lemma 24 (Weidmann [21]) Let $M$ be a graph manifold of Heegaard genus 3 which is not Seifert fibered and has 2-generated fundamental group. Then the following hold:

(1) $\quad M=M_{1} \cup_{T} M_{2}$ where $M_{1}$ is fibered over Mö or Mö $(p)$ and $M_{2}$ is fibered over $D(2,2 l+1)$.

(2) The intersection number of the the fibres $f_{1}$ and $f_{2}$ on $T$ is $\Delta\left(f_{1}, f_{2}\right)= \pm 1$.

(3) The pair $\left(M_{1}, f_{2}\right)$ is not the exterior of a 1-bridge knot in a lens space with $f_{2}$ being a meridian.

(4) The pair $\left(M_{2}, f_{1}\right)$ is not the exterior of a 2-bridge knot in $S^{3}$ with $f_{1}$ being a meridian.

Proof of Proposition 23 If $\pi_{1}(M)$ admits an inversion it follows from Proposition 20 that it is induced by an involution $\tau$, unique up to conjugacy and which preserves each Seifert piece and their Seifert fibrations. We distinguish the cases where the involution $\tau$ has fixed points on the JSJ-splitting torus $T$ and where it does not.

Case 1: $\operatorname{Fix}(\tau) \cap T \neq \varnothing$ Then $\tau$ induces an hyperelliptic involution on $T$ and the fixed point set of $\tau$ meets the splitting torus $T$ in 4 points. The quotient spaces $M_{1} / \tau$ and $M_{2} / \tau$ together with the image of the fixed point set of $\tau$ are two non-rational Montesinos tangles (see Bonahon and Siebenmann [9]): we use notations analogous to the ones used for Montesinos links in Boileau and Zieschang [7, Section 2] or Burde and Zieschang [10, Chapter 12]

$B_{1}=M_{1} / \tau=B(-1 ; \beta / p)$ where $\beta / p$ is the type of the singular fiber of order $p$ of $M_{1}$, see Figure 1 . 


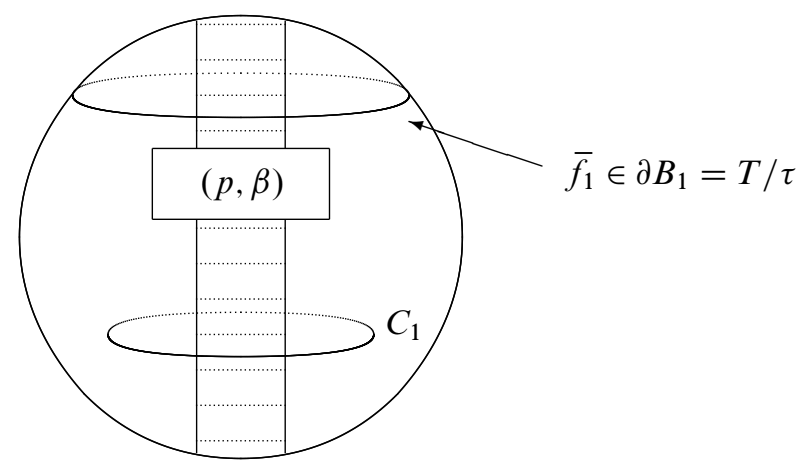

Figure 1: $B_{1}=B(-1 \mid \beta / p)$

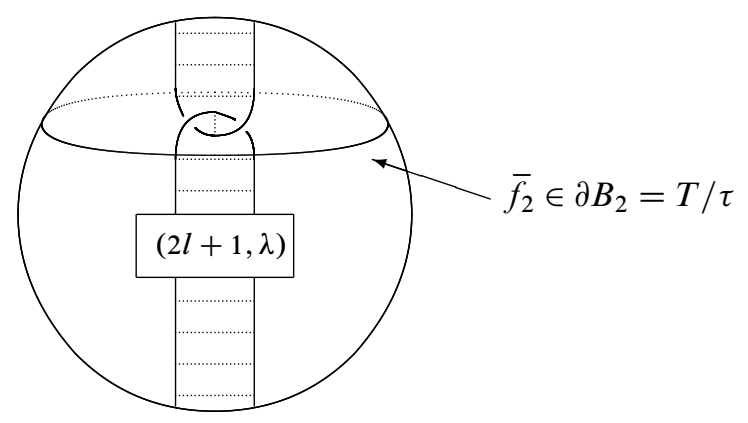

Figure 2: $B_{2}=B(0 \mid 1 / 2, \lambda /(2 l+1))$

$B_{2}=M_{2} / \tau=B(0 ; 1 / 2, \lambda / 2 l+1)$ where $\lambda / 2 l+1$ is the type of the singular fiber of order $2 l+1$ of $M_{2}$, see Figure 2 .

The manifold $M$ is a 2 -fold covering of $S^{3}$ branched along a so called Conway algebraic link $L$ obtained by gluing the two tangles $B_{1}$ and $B_{2}$ along their boundary (see Bonahon and Siebenmann [9]). The link $L=K \cup C_{1}$, where $K$ is a 2-bridge knot or link by Proposition 20, and $C_{1}$ is an unknotted component of the Montesinos tangle $B_{1}$ which corresponds to a $S^{1}$-fiber of the orbifold Seifert fibration of $B_{1}$ obtained by quotienting the Seifert fibration of $M_{1}$ (see Figure 1 and Montesinos [15; 16]). We remark that when forgetting the component $C_{1}$, the Montesinos tangle $B_{1}$ becomes a rational tangle $B_{1}^{\prime}$ of type $\beta / p \in \mathbb{Q} / \mathbb{Z}$ with the orbifold Seifert fibration induced from the one of $B_{1}$. The rational tangle $B_{1}^{\prime}$ contains a properly embedded meridian disk $\left(\Delta_{1}, \partial \Delta_{1}\right) \hookrightarrow\left(B_{1}^{\prime}, \partial B_{1}^{\prime}\right)$ which separates its two strands. Let $\delta_{1}=\partial \Delta_{1}$ be the simple closed curve on the pillow $\partial B_{1}^{\prime}=\partial B_{1}$. In order to obtain a 2-bridge knot or link $K$ when gluing the rational tangle $B_{1}^{\prime}$ to the Montesinos tangle $B_{2}$, one needs that the orbifold Seifert fibration of $B_{2}$ (inherited from the Seifert fibration of 
$M_{2}$ ) extends to an orbifold Seifert fibration on the tangle $B_{1}^{\prime}$ without singular fiber. Otherwise the 2-fold branched covering of $K$ obtained by Dehn filling $M_{2}$ would have three singular fibers and could not be a Lens space.

Let $\overline{f_{1}}$ and $\overline{f_{2}}$ be the images on $\partial B_{1}^{\prime}=\partial B_{2}$ of the fibers $f_{1} \subset \partial M_{1}=T$ and $f_{2} \subset \partial M_{2}=T$. We say that two simple closed curves on the pillow $\partial B_{1}^{\prime}=\partial B_{2}$ are dual if they are the images of two simple closed curves on the torus $T$ which are dual, that is, meets in one point. Since $\Delta\left(f_{1}, f_{2}\right)= \pm 1$ it follows that $\bar{f}_{1}$ and $\bar{f}_{2}$ are dual on the pillow $\partial B_{1}^{\prime}=\partial B_{2}$, and thus the orbifold Seifert fibration of the pillow $\partial B_{1}^{\prime}=\partial B_{2}$ with fiber $\bar{f}_{2}$ extends to an orbifold Seifert fibration of the tangle $B_{1}^{\prime}$ with a singular fiber of order $\beta$. Hence $\beta= \pm 1$. In this case $\bar{f}_{2}$ is also dual to the curve $\delta_{1} \subset \partial B_{1}^{\prime}=\partial B_{2}$. Therefore, up to orientation, there is at most one possibility for choosing $\bar{f}_{2}$ on the pillow $\partial B_{1}^{\prime}=\partial B_{2}$, and thus for choosing $f_{2}$ on $T$.

The condition $\beta= \pm 1$ is equivalent for $M_{1}$ to be the exterior of a one-bridge knot in a Lens space by Weidmann [21, Lemma 1]. Therefore the existence of an inversion on $\pi_{1}(M)$ implies that $M_{1}$ is the exterior of a one-bridge knot in a lens space and that, up to orientation of $M_{1}$, there is at most one possible choice for the fiber $f_{2}$ on $T=\partial M_{1}$. Kobayashi's construction of Heegaard genus two graph-manifolds shows that this happens precisely when $f_{2}$ is the meridian of the knot space in the Lens space, which is the excluded case.

Case 2: $\operatorname{Fix}(\tau) \cap T=\varnothing \quad$ In this case the quotient spaces $V_{1}=M_{1} / \tau$ and $V_{2}=M_{2} / \tau$ are Seifert fibered 3-orbifolds with boundary the torus $T / \tau$ such that $V_{1} \cup V_{2}=S^{3}$.

The action induced by $\tau$ on the base $D(2,2 l+1)$ of $M_{2}$ must be the identity, hence the restriction of $\tau$ to $M_{2}$ is the $\mathbb{Z} / 2 \mathbb{Z}$ action embedded into the $S^{1}$-action defining the Seifert fibration on $M_{2}$. In particular the exceptional fibre of order 2 of $M_{2}$ is fixed pointwise by $\tau$. Thus $V_{2}$ is a Seifert fibered solid torus with base $D(2 l+1)$ and the component $K_{2} \subset V_{2}$ of the branching link L is a regular fiber in this Seifert fibered solid torus, by Seifert [19, Section 14].

Since the quotient $M_{1} / \tau$ embeds in $S^{3}$ with boundary a torus, the action induced by $\tau$ on the base Mö or $\operatorname{Mö}(p)$ cannot be the identity and cannot have fixed point on the boundary, hence it is the reflection through the core of the underlying Möbius strip, where the singular point of order $p$ is assumed to lie on this core.

To understand the restriction of the involution $\tau$ to $M_{1}$ we first consider the case where $M_{1}$ is the twisted $I$-bundle over the Klein bottle, that is, the base is a Möbius strip. In this case the quotient $V_{1}=M_{1} / \tau$ is the Euclidean 3-orbifold which is an orientable bundle (in the orbifold sense) over a Möbius strip with silvered boundary, where a boundary is called silvered if it corresponds to a reflection axis (see, for example, 
Boileau and Porti [4, Chapter 4]). The underlying space is a solid torus which is a neighborhood of the Möbius strip and the branching locus $K_{1}$ is the boundary of the Möbius strip. The quotient $V_{1}$ is a Seifert 3-orbifold: each meridian disk of the solid torus $V_{1}$ is fibered by concentric circles together with an arc joining the two intersection points of the branching locus with the meridian disk.

To understand the restriction of the involution $\tau$ to $M_{1}$ when the base is $\operatorname{Mo}(p)$, we have just to pull out a tubular neighborhood of a $\tau$-invariant regular fiber in the previous example and glue it back to insert a singular fiber of type $\beta / p$. In the quotient $V_{1}$ it corresponds to pulling out a tubular neighborhood of a fiber which is an interval with silvered boundary (that is, a non-separating arc of the Möbius strip bounded by the branching locus $K_{1}$ ) and inserting a rational tangle of type $\beta / p$. The quotient $V_{1}$ is still a Seifert 3-orbifold with underlying space a solid torus $V_{1}$ and whose boundary $\bar{T}=T / \tau$ is fibered by the boundaries of meridian disks of $V_{1}$, see Figure 3 .

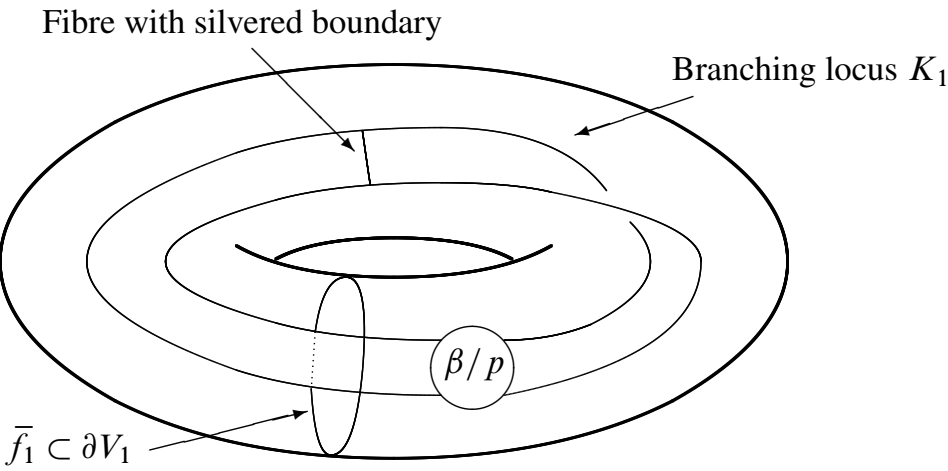

Figure 3: The Seifert fibered orbifold $V_{1}$

Let $L=K_{1} \cup K_{2}$ be the branching link $L \subset S^{3}$, with $K_{1} \subset V_{1}$ and $K_{2} \subset V_{2}$. Let $\Delta_{1}$ and $\Delta_{2}$ be meridian disks of the solid tori $V_{1}$ and $V_{2}$ respectively. Since $V_{1} \cup V_{2}$ is $S^{3}$, their boundaries verify: $\Delta\left(\partial \Delta_{1}, \partial \Delta_{2}\right)= \pm 1$. Thus $\partial \Delta_{1}$ and $\partial \Delta_{2}$ form a base of $H^{1}(\bar{T}, \mathbb{Z})$ for the torus quotient $\bar{T}=T / \tau$. Let $\bar{f}_{1}$ and $\bar{f}_{2}$ be the images on $\bar{T}$ of the fibres $f_{1}$ and $f_{2}$ on $T$.

Since $K_{2}$ is isotopic to the regular fibre $\bar{f}_{2}$ of the fibered solid torus $V_{2}$, it can be pushed onto $\bar{T}=\partial V_{1}=\partial V_{2}$. Moreover $K_{2}=\bar{f}_{2}$ will meet $f_{1}=\partial \Delta_{1}$ in one point, since $f_{2}$ meets $f_{1}$ geometrically in one point on $T=\partial M_{1}=\partial M_{2}$. Therefore $K_{2}$ is unknotted in $S^{3}$ and the 2-fold covering of $V_{2}$ branched over $K_{2}$ embeds into the 2-fold branched covering of $S^{3}$ branched along $K_{2}$, which is still $S^{3}$. Hence $M_{2}$ is the exterior of a torus knot of type $(2,2 l+1)$, which is a 2 -bridge knot. 
Let $\mu_{2}$ be the meridian curve on $\partial M_{2}$ when $M_{2}$ is considered as a knot exterior in $S^{3}$. The Seifert surface $F_{2}$ of the fibred torus knot exterior $M_{2}$ verifies that $\Delta\left(\mu_{2}, \partial F_{2}\right)=1$ and $\Delta\left(\partial F_{2}, f_{2}\right)= \pm 2(2 l+1)$. Moreover $\partial F_{2}$ projects to the boundary $\partial \Delta_{2}$ of the meridian disk of $V_{2}$.

As the fiber $\bar{f}_{1}=\partial \Delta_{1}$ meets $\partial \Delta_{2}$ in one point, we have $\left|\Delta\left(f_{1}, \partial F_{2}\right)\right| \leq 2$. Therefore we have shown that:

(1) $\left|\Delta\left(\partial F_{2}, f_{2}\right)\right|=2(2 l+1)$

(2) $\left|\Delta\left(f_{1}, \partial F_{2}\right)\right| \leq 2$

(3) $\left|\Delta\left(f_{1}, f_{2}\right)\right|=1$

By (1), $f_{2}=\partial F_{2} \pm 2(2 l+1) \mu_{2}$ on $\partial M_{2}=T$.

By (2) and (3) $2(2 l+1)\left|\Delta\left(\mu_{2}, f_{1}\right)\right| \leq 3$, and hence $\Delta\left(\mu_{2}, f_{1}\right)=0$. This would imply that $\mu_{2}$ and $f_{1}$ are isotopic on $T=\partial M_{2}$ contradicting our hypothesis.

Another argument to get a contradiction would be to show that, after pushing $K_{2}$ into the unknotted solid torus $V_{1}, L$ has at most 3 bridges using the method in Boileau and Zieschang [7].

\section{References}

[1] C C Adams, Hyperbolic 3-manifolds with two generators, Comm. Anal. Geom. 4 (1996) 181-206 MR1393562

[2] M A Armstrong, The fundamental group of the orbit space of a discontinuous group, Proc. Cambridge Philos. Soc. 64 (1968) 299-301 MR0221488

[3] J S Birman, HM Hilden, Heegaard splittings of branched coverings of $S^{3}$, Trans. Amer. Math. Soc. 213 (1975) 315-352 MR0380765

[4] M Boileau, J Porti, Geometrization of 3-orbifolds of cyclic type, Astérisque (2001) 208 MR1844891 Appendix A by Michael Heusener and Porti

[5] M Boileau, R Weidmann, The structure of 3-manifolds with two-generated fundamental group, Topology 44 (2005) 283-320 MR2114709

[6] M Boileau, H Zieschang, Heegaard genus of closed orientable Seifert 3-manifolds, Invent. Math. 76 (1984) 455-468 MR746538

[7] M Boileau, H Zieschang, Nombre de ponts et générateurs méridiens des entrelacs de Montesinos, Comment. Math. Helv. 60 (1985) 270-279 MR800006

[8] M Boileau, B Zimmermann, The $\pi$-orbifold group of a link, Math. Z. 200 (1989) 187-208 MR978294 
[9] F Bonahon, S Siebenmann, Geometric splittings of classical knots and the algebraic knots of Conway (to appear)

[10] G Burde, H Zieschang, Knots, de Gruyter Studies in Mathematics 5, Walter de Gruyter \& Co., Berlin (1985) MR808776

[11] B Fine, G Rosenberger, Classification of all generating pairs of two generator Fuchsian groups, from: "Groups '93 Galway/St. Andrews, Vol. 1 (Galway, 1993)”, London Math. Soc. Lecture Note Ser. 211, Cambridge Univ. Press, Cambridge (1995) 205-232 MR1342792

[12] I Kapovich, R Weidmann, Two-generated groups acting on trees, Arch. Math. (Basel) 73 (1999) 172-181 MR1705011

[13] S P Kerckhoff, The Nielsen realization problem, Ann. of Math. (2) 117 (1983) 235-265 MR690845

[14] R Kirby, Problems in low-dimensional topology, from: "Geometric topology (Athens, GA, 1993)”, AMS/IP Stud. Adv. Math. 2, Amer. Math. Soc., Providence, RI (1997) 35-473 MR1470751

[15] J M Montesinos, Seifert manifolds that are ramified two-sheeted cyclic coverings, Bol. Soc. Mat. Mexicana (2) 18 (1973) 1-32 MR0341467

[16] J M Montesinos, Classical tessellations and three-manifolds, Universitext, Springer, Berlin (1987) MR915761

[17] N Peczynski, G Rosenberger, H Zieschang, Über Erzeugende ebener diskontinuierlicher Gruppen, Invent. Math. 29 (1975) 161-180 MR0382457

[18] M Rost, H Zieschang, Meridional generators and plat presentations of torus links, J. London Math. Soc. (2) 35 (1987) 551-562 MR889376

[19] H Seifert, Topologie Dreidimensionaler Gefaserter Räume, Acta Math. 60 (1933) 147-238 MR1555366

[20] W P Thurston, The Geometry and Topology of Three-Manifolds, Princeton Univ. Math. Dept. Notes (1979)

[21] R Weidmann, Some 3-manifolds with 2-generated fundamental group, Arch. Math. (Basel) 81 (2003) 589-595 MR2029721

[22] H Zieschang, Über die Nielsensche Kürzungsmethode in freien Produkten mit Amalgam, Invent. Math. 10 (1970) 4-37 MR0263929

[23] H Zieschang, Finite groups of mapping classes of surfaces, Lecture Notes in Mathematics 875, Springer, Berlin (1981) MR643627

[24] H Zieschang, B Zimmermann, Über Erweiterungen von $\mathbb{Z}$ und $\mathbb{Z}_{2} * \mathbb{Z}_{2}$ durch nichteuklidische kristallographische Gruppen, Math. Ann. 259 (1982) 29-51 MR656650

[25] B Zimmermann, Periodische Homöomorphismen Seifertscher Faserräume, Math. Z. 166 (1979) 289-297 MR526469

Geometry ${ }^{6}$ Topology Monographs, Volume 14 (2008) 
[26] B Zimmermann, Das Nielsensche Realisierungsproblem für hinreichend große 3Mannigfaltigkeiten, Math. Z. 180 (1982) 349-359 MR664520

[27] B Zimmermann, Finite group actions on Haken 3-manifolds, Quart. J. Math. Oxford Ser. (2) 37 (1986) 499-511 MR868625

Laboratoire Émile Picard, CNRS UMR 5580, Université Paul Sabatier

118 Route de Narbonne, F-31062 Toulouse Cedex 4, France

Department of Mathematics and the Maxwell Institute of Mathematical Sciences

Heriot-Watt University, Riccarton, Edinburgh, EH14 4AS, Scotland

boileau@picard.ups-tlse.fr, R.Weidmann@ma.hw.ac.uk

Received: 21 October 2006

Geometry $\mathcal{E}_{\mathcal{G}}$ Topology Monographs, Volume 14 (2008) 